Check for updates

Cite this: Nanoscale Adv., 2019, 1, 973 Accepted 28th December 2018

DOI: $10.1039 / c 8 n a 00345 a$

rsc.li/nanoscale-advances

\section{PU nanocomposites from bifunctional nanoparticles: impact of liquid interphase on mechanical properties $\uparrow$}

\author{
Cibele Carneiro Pessan, (iD a Bruno Henrique Ramos de Lima (iD ${ }^{b}$ \\ and Edson Roberto Leite (D) *cd
}

The $\mathrm{Fe}_{3} \mathrm{O}_{4}$ aPoly $(1,4-$ butanediol $) /$ polyurethane nanocomposite is a highly interphase-dependable material with unique characteristics. Firstly, the nanoparticle's organic shell allows simple fabrication of very well dispersed nanocomposites and the incorporation of extremely high amounts of nanoparticles (NP) into the polymer matrix. Secondly, both chemical and physical aspects of the nanoparticles determine the material's mechanical behavior. The chemical functionality of the organic layer - free hydroxyl groups at the end of the tethered chains - ensures the material's stiffening through covalent bonds with the matrix, while being at molten state provides high flexibility and deformability yet maintaining mechanical resistance. As a result, nanocomposites at the low concentration region show increased elastic modulus and tensile strength and slight increase in total strain, while highly concentrated nanocomposites show reduction of elastic modulus and tensile strength and roughly double the total strain. The combination of the chemical and physical functionalities ensures high compatibility between nanoparticles and matrix and allows the production of highly concentrated - above $90 \%$ in weight - nanocomposites as a cohesive and flexible material, instead of a brittle wafer. This bifunctionality effect is unprecedented and the results open a wide range of new possibilities in the tailoring of functional nanomaterials for all sorts of applications in materials science.

One of the main challenges regarding the processing of nanocomposites with polymeric matrix is achieving satisfactory dispersion of the nanometric phase, as well as a suitable interface between the polymer phase and the nanoparticle. For

${ }^{a}$ Materials Engineering Department, Federal University of São Carlos (UFSCar), 13565-905, São Carlos SP, Brazil. E-mail: cibele.pessan@ppgcem.ufscar.br

${ }^{b} n$ Chemi - Engenharia de Materiais LTDA, Rua Alfredo Lopes, 1717, sala d11, 13560460, São Carlos SP, Brazil. E-mail: bruno.lima@nchemi.com

${ }^{c}$ Chemistry Department, Federal University of São Carlos (UFSCar), 13565-905, São Carlos SP, Brazil

${ }^{d}$ Brazilian Nanotechnology National Laboratory (LNNano), Brazilian Center for Research in Energy and Materials (CNPEM), Campinas SP, Brazil. E-mail: edson. leite@lnnano.cnpem.br

$\dagger$ Electronic supplementary information (ESI) available. See DOI: 10.1039/c8na00345a instance, a good interface, or good compatibility between the filler and the matrix can result in an efficient transference of mechanical stresses between the composite components, which will control the thermo-mechanical behavior of the nanocomposite. However, without proper dispersion of the nanometric phase into the matrix, the true potential of nanosized particles to modify the mechanical behavior of the polymeric materials is lost. ${ }^{1-9}$

First attempts to provide a suitable nanoparticle dispersion were focused on tweaking the processing parameters in search of higher dispersion levels, such as high shear mixing processes or quenching of solvent solutions. These approaches have achieved a limited success, mostly related to the difficulty in increasing the volume of fillers incorporated into the polymeric matrix or scale-up likelihood. On the other hand, there are dispersion strategies based on the nanoparticle's shape and chemistry, such as steric stabilization which is sustained by the nanoparticle functionalization.,.$^{2,4,9-12}$

There are many designations for functionalized nanoparticles, such as hybrid nanoparticles, hybrid organic-inorganic nanoparticles, hybrid core-shell nanoparticles, polymernanoparticle composite, molecular composite, polymergrafted nanoparticle and hairy nanoparticles. This type of nanoparticle has organic molecules adsorbed or covalently attached to the nanoparticle's surface, thus changing its volume and chemical properties. Depending on the chemical nature of these molecules, the nanoparticle can become hydrophobic or hydrophilic or acquire the properties of a reactive polyelectrolyte. , $^{\mathbf{4}, 9,13-18}$

It is well described in the literature that organic molecules bonded to a nanoparticle's surface prevent its agglomeration by steric stabilization, enabling better dispersion of nanoparticles in the medium. ${ }^{19-21}$ In liquid solvents it is possible to create very stable colloidal dispersion as long as the solvent and the organic layer are compatible, i.e., the solvent and the capping ligand having similar solubility parameters. Similar concept can be applied to the dispersion of functionalized nanoparticles within a polymer matrix: if the nanoparticle's organic shell is 
compatible with the matrix molecules, it will exhibit a more extended conformation around the core which increases the distance between cores and prevents agglomeration. .,19,22,23 $^{\mathbf{4}}$

In general, nanoparticles are functionalized with low molecular weight organic compound (smaller than $350 \mathrm{~g}$ $\mathrm{mol}^{-1}$ ). However, molecules with high molecular weight, in special polyols and polymers, have been successfully attached to the surface of inorganic nanoparticles (ranging from 1000 to $30000 \mathrm{~g} \mathrm{~mol}^{-1}$ ). Hybrid nanoparticles formed by organic molecules with high molecular weight can exhibit a more complex interphase region in the polymeric nanocomposite, bringing novel mechanical, thermic and electronic behavior to the materials. Actually, we can tailor a nanocomposite where its property will be controlled by the interphase, which can bring unusual combination of properties. ${ }^{6,12,19,24}$

In this paper we describe a novel nanocomposite system, using a nanoparticle functionalized with a high molecular weight organic compound, where the mechanical behavior is fully controlled by the interphase. Here, a highly interphasedependable hybrid nanocomposite, constituted by polyurethane matrix and functionalized magnetite $\left(\mathrm{Fe}_{3} \mathrm{O}_{4}\right)$ nanoparticles, which have an organic capping layer of poly(1,4butanediol) (molecular weight of $1000 \mathrm{~g} \mathrm{~mol}^{-1}$ ), was prepared by colloidal process. Firstly, the use of poly(1,4-butanediol) $\left(\mathrm{PB}_{1000}\right)$ as the nanoparticles' organic shell is interesting due to its very high affinity with the polyurethane (PU) system. In this nanocomposite the matrix must act as a good solvent for the nanoparticles, which will ensure good nanoparticle dispersion. Secondly, the NP's organic shell presents hydroxyl groups at the end of the polymeric chains, which can react with the free isocyanate (NCO) groups present in the PU prepolymer system (characterized by the excess of isocyanate). Since the OH groups available in the nanoparticles participate in the PU's curing reaction, the result is a strong interaction between matrix and nanoparticles through covalent bonds at the urethane functional groups formation. Finally, since the $\mathrm{PB}_{1000}$ molecules are at molten state at room temperature, the NP's organic shell exhibits very high mobility and consequently provides flexibility to the final material, as well as participates in mechanical entanglements with polymer matrix. Therefore, for this specific system, there are three phases: inorganic nanoparticle core, molten interphase and matrix. ${ }^{25}$

The nanocomposites (PU and functionalized nanoparticles) were produced by mixing and solvent evaporation process. We studied two ranges of concentration: low concentration nanocomposites, ranging from $0.05 \%$ wt to $5.0 \%$ wt of nanoparticles, and highly concentrated nanocomposites that ranged from $60 \%$ wt to $90 \% w t$ of nanoparticles. Here, the different nanocomposite formulations are named from PU-0.05\% throughout PU-90\%, and the percentages in the name refer to the nanoparticle content (in wt\%) in each composition. Non-filled formulations were also prepared to simulate the effects of poly(1,4-butanediol) in the PU matrix. The formulations are named Blend-0.05\% throughout Blend-2.5\%, and the percentages in the name refer to the corresponding nanocomposite formulation, which presents the same quantity of $\mathrm{PB}_{1000}$ in its composition (for more details, see ESI Table $\mathrm{S} 1 \uparrow$ that presents all formulated compositions).

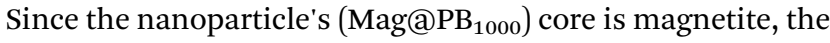
nanoparticle colloidal dispersion in tetrahydrofuran (THF) has a dark crimson color and behaves as a ferrofluidic liquid under an applied magnetic field. Thermogravimetric analysis (TGA) of dried nanoparticles shows that the inorganic cores represent $60.5 \%$ of the nanoparticles' weight, while the organic shell ads up to the remaining $39.5 \%$ wt (for details see ESI Fig. S1†). The main results regarding the nanoparticles characterization are shown in Fig. 1.

Transmission Electron Microscopy (TEM) image pictured in Fig. 1a shows freestanding nanoparticles with cores formed by a single crystallite magnetite domain, which have an average diameter of $5.7 \pm 1.5 \mathrm{~nm}$ (for details see High Resolution TEM (HR-TEM) image in the inset in Fig. 1a). ${ }^{19}$

The analysis by Fourier-Transform Infrared spectroscopy (FTIR), shown in Fig. 1b, present four key identifying peaks related to unsaturated primary alcohol. The first is the broad peak between 3100 and $3600 \mathrm{~cm}^{-1}$, indicative of exchangeable protons, typical of alcohols, amine, amide or carboxylic acid groups. The second signal of interest is a pair of peaks, located at 2935 and $2861 \mathrm{~cm}^{-1}$. When the second has greater intensity than the first peak, it is an indicative that the $2935 \mathrm{~cm}^{-1}$ peak is related to the $\mathrm{R}-\mathrm{CH}_{2}-\mathrm{OH}$ asymmetric stretching. Finally, the peak at $1365 \mathrm{~cm}^{-1}$ is related to the angular deformation of $\mathrm{OH}$ end group and the $1110 \mathrm{~cm}^{-1}$ is linked to the axial deformation of a $\mathrm{C}-\mathrm{O}$ bond in alcohols. All these results together confirm the organic shell's molecules are hydroxyl group terminated.

The nanoparticles were subjected to heating and cooling cycles in Differential Scanning Calorimetry (DSC) analysis in order to evaluate their thermal behavior. The resulting curves of first and second temperature cycles can be seen in Fig. 1c. The first and second cycle results coincide (noticeable in Fig. S3, available in ESI $\dagger$ ), which means no organic or volatile compound was released nor did the molecules carry any thermal history from its synthesis process.
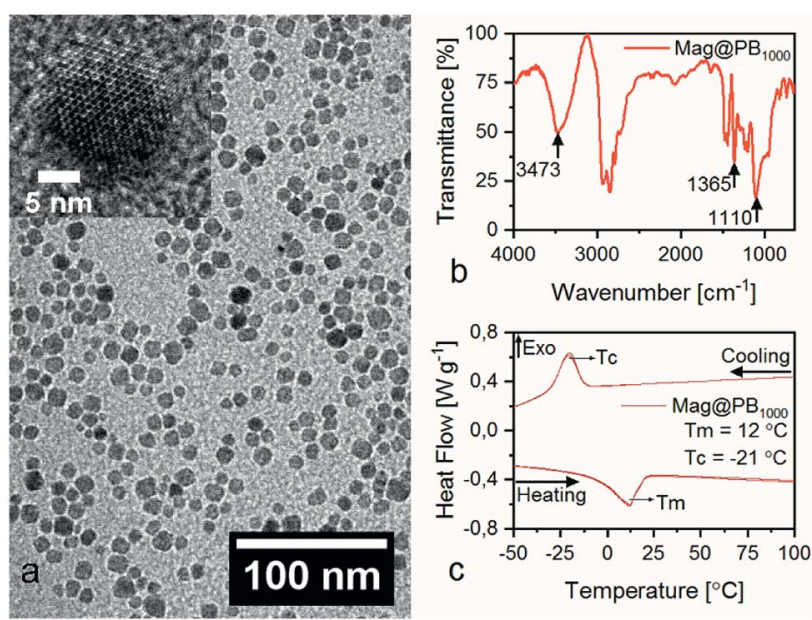

Fig. 1 (a) TEM image, the inset shoes HR-TEM image of a freestanding NP. (b) FTIR analysis and (c) DSC heat flow analysis of Mag@PB 1000 nanoparticles. 
The curves present reversible first order transformations, one at heating and another at cooling stage, that resemble the commercial Terathane ${ }_{1000}{ }^{\circledR}$ 's thermal behavior (available in ESI Fig. S2 $\dagger$ ), but with broader and less intense peaks. Consequently, the endothermic peak at $12{ }^{\circ} \mathrm{C}$ during heating stage and the exothermic peak at $-21^{\circ} \mathrm{C}$ during cooling stage refer to melting and crystallization transformations of the $\mathrm{PB}_{1000}$ present in the nanoparticles, respectively. To our knowledge, this phenomenon was reported in few studies, in which low molecular weight fatty acids (stearic, palmitic and erucic) attached to $\gamma$-alumina presented such endotherms. ${ }^{12}$ However, it is the first time such transition is reported at ambient temperature and without considerable shift from the pristine material's melting temperature. This means the organic shell attached to the $\mathrm{Fe}_{3} \mathrm{O}_{4}$ inorganic core is at its molten state at room temperature and consequently the organic layer presents itself in a random coil conformation, which results in high molecular mobility and entanglement between chains. Therefore, the $\mathrm{Mag} @ \mathrm{~PB}_{1000}$ are bifunctional nanoparticles whose solid inorganic core is covalently bonded to a molten hydroxyl group terminated organic shell..$^{6,10,12,22,23}$

Following the nanoparticles' synthesis, the nanocomposite and blend films were produced through mixing and solvent evaporation. As the nanoparticles' concentration rises, the materials' color becomes more intense and darker and its appearance shifts from translucent and glossy to an opaque and matte finish (film samples of all formulations are presented in Fig. S4†).

Fig. 2 pictures electron microscopy images of two nanocomposites (Mag@PB $\mathrm{PB}_{1000} / \mathrm{PU}$ ) formulations. The TEM image of PU-5.0\%, shown in Fig. 2a, shows evenly dispersed nanoparticles, with no agglomerates or preferred orientation within the PU matrix (a higher magnification inset image is presented for more detailed view). Differently, PU-90\% formulation, pictured in Scanning Electron Microscopy (SEM) image, shown in Fig. 2b, is a highly concentrated material, in which the nanoparticles touch each other (for details, see TEM image in the inset in Fig. 2b). The SEM image captures the moment thermal degradation of a section of the sample occurs. Between two pores, a portion of the PU matrix is slowly elongating and thinning, until inevitable rupture. In this site, despite the small distance and probable mechanical entanglements the nanoparticles may have with each other, they still interact strongly

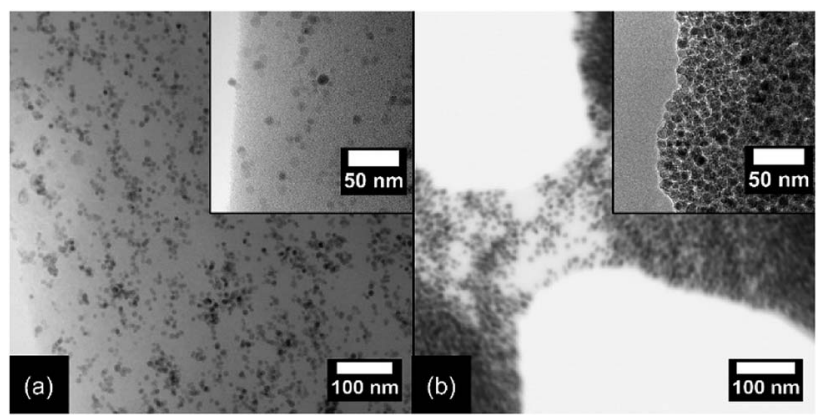

Fig. 2 TEM image at different magnifications for (a) the PU-5.0\% composition and (b) PU-90\% composition. with the matrix and dislocated themselves according to the matrix' deformation.

The main results for $\mathrm{Mag} @ \mathrm{~PB}_{1000} / \mathrm{PU}$ nanocomposites are shown in Fig. 3. DSC heating curves of the low concentration nanocomposites are presented in Fig. 3a, in which a second order transformation is visible: glass transition. In the image, the gray area sets the limit between beginning and end of the transition (onset and endset), the yellow area presents the region in which all the glass transition temperatures $\left(T_{\mathrm{g}}\right)$ are located (between the minimum and maximum $T_{\mathrm{g}}$ values of all nanocomposites) and the black vertical line indicates the average nanocomposite $T_{\mathrm{g}}$ value. As seen in Fig. 3a, the low concentration nanocomposites present similar thermal behavior as the PU formulation and the $T_{\mathrm{g}}$ values, which are presented in Table $\mathrm{S} 2, \dagger$ are in the same temperature range. The non-filled formulations also exhibit the same thermal behavior and same range for $T_{\mathrm{g}}$ values as the pristine PU and the corresponding nanocomposites formulations (for more details on $T_{\mathrm{g}}$ values and the thermal behavior of the blend formulations, see ESI Fig. S5a and Table S2 $\dagger$ ). This indicates that the presence of the hybrid nanoparticles' rigid core did not contribute significantly to the formulations' stiffening, but rather the presence of the hydroxyl groups available at the nanoparticles' organic shell (or the free $\mathrm{PB}_{1000}$ molecules in the case of the non-filled formulations).

On the other hand, the DSC heating and cooling curves of the highly concentrated formulations, shown in Fig. 3b, show that, as the nanoparticle weight fraction increases, a reversible first order transformation grows stronger and more alike to the behavior seen for $\mathrm{Mag} \mathrm{PB}_{1000}$. There is an endothermic peak during heating stage and exothermic peak during cooling stage, that become more visible for the most concentrated formulation, PU-90\%. Comparing the nanocomposites' curves to the NP's (as shown in Fig. S5b†), it is seen that the peaks refer to the melting and crystallization of the $\mathrm{PB}_{1000}$ (as previously discussed), present in the nanocomposites' organic shell.

So, the low concentration nanocomposites and blend formulations present the same thermal behavior as the pristine PU, whereas the highly concentrated compositions reflect the nanoparticles' thermal behavior - specifically the thermal behavior of its organic shell. This means that, since at room temperature the organic shell is molten, PU-60\%, PU-70\% and PU-90\% formulations present roughly $25 \%, 28 \%$ and $35 \%$ of their total mass in molten state, respectively.

The tensile stress-strain curves obtained for the nanocomposites and the reference composition (PU) are shown in Fig. 3c, except for the PU-90\% formulation, which presented erroneous results due to high porosity. The mechanical behavior of the nanocomposites is that of rubber like materials, in which there are three distinct zones in the stress-strain curve: the first is a steep region of the stress-strain curve that relates to the crystallinity and stiffness of the material. Following, is a less steep region (that can be short or elongated) which is related to the deformation of soft amorphous domains and alignment of hard domains. Finally, the curve ramps up to the final segment, that is related to the stretching of soft and hard domains of the rubbery material. ${ }^{5,26}$ 

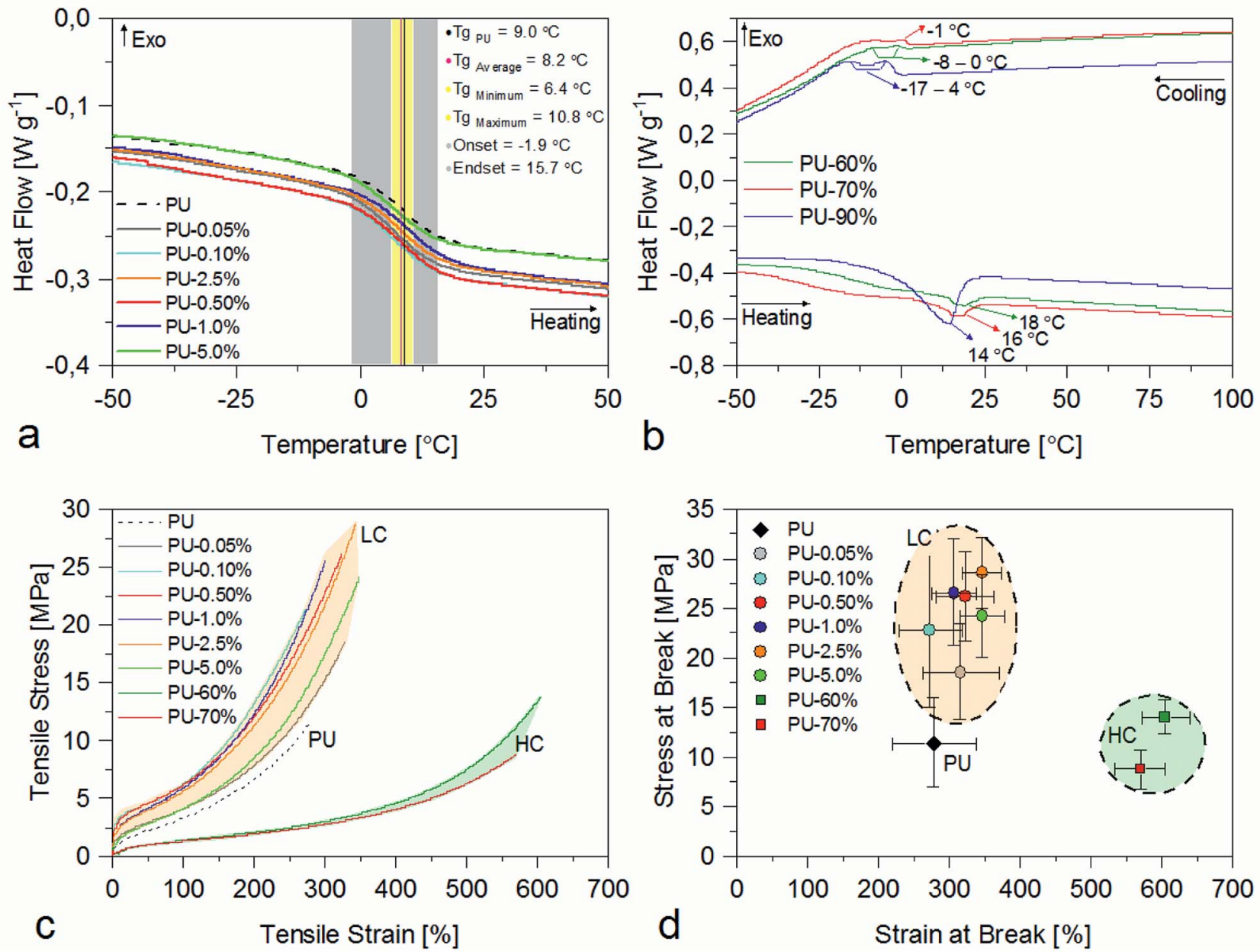

Fig. 3 (a) DSC heat flow curves for low concentration nanocomposites compositions and (b) for the highly concentrated Mag@PB $1000 / \mathrm{PU}$

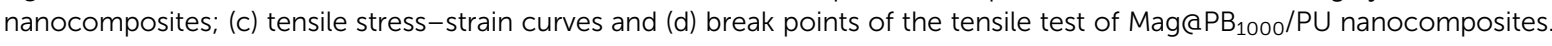

As seen in Fig. 3c, the addition of NPs doesn't change the behavioral profile of the curve, however we observe changes in elastic modulus and elongation, compared to the pristine PU. For the low concentration compositions, the curves become steeper and slightly more elongated, and for the higher weight fractions of NPs, the materials present lower elastic modulus while doubling in elongation.

As presented in Table 1 - which displays values for elastic modulus, stress at rupture and strain at rupture for all formulations - there is increase in Young's modulus, tensile strength and total deformation for the nanocomposites, in exception to the highly concentrated formulations, that exhibit lower Young's modulus and little change in tensile strength.

The nanocomposites presented here differ from typical nanocomposites, since tensile test results show that it occurs simultaneous strengthening and increasing of total deformation of the material. The strengthening of low concentration nanocomposites is noticeable by the increase of Young's modulus value and overall stress response and tensile strength, but the anomaly is present for the highly concentrated compositions, that exhibit lower Young's modulus and much
Table 1 Values of stress and strain at break and instant elastic modulus of nanocomposites and non-filled formulations

\begin{tabular}{llll}
\hline & $\begin{array}{l}\text { Stress at break } \\
\text { Formulation }\end{array}$ & $\begin{array}{l}\text { Strain at break } \\
\text { point }[\mathrm{MPa}]\end{array}$ & $\begin{array}{l}\text { Elastic modulus } \\
{[\mathrm{MPa}]}\end{array}$ \\
\hline
\end{tabular}

Polyurethane reference composition

$\begin{array}{llll}\text { PU } & 13 \pm 2 & 297 \pm 22 & 7 \pm 2\end{array}$

Low concentration nanocomposite formulations

$\begin{array}{llll}\text { PU- } 0.05 \% & 17 \pm 5 & 330 \pm 32 & 11 \pm 3\end{array}$

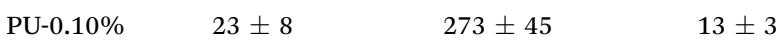

$\begin{array}{llll}\text { PU- } 0.50 \% & 26 \pm 5 & 323 \pm 41 & 13 \pm 2\end{array}$

$\begin{array}{lllr}\text { PU-1.0\% } & 28 \pm 4 & 308 \pm 31 & 16 \pm 2\end{array}$

$\begin{array}{llll}\text { PU-2.5\% } & 30 \pm 1 & 354 \pm 16 & 15.6 \pm 0.7\end{array}$

$\begin{array}{llll}\text { PU-5.0\% } & 24 \pm 3 & 347 \pm 31 & 14 \pm 1\end{array}$

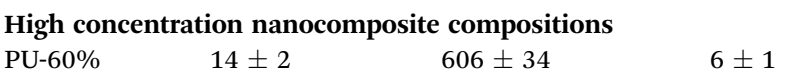

$\begin{array}{rrrr}\text { PU-60\% } & 14 \pm 2 & 606 \pm 34 & 6 \pm 1 \\ \text { PU-70\% } & 9 \pm 2 & 570 \pm 36 & 3.4 \pm 0.9\end{array}$

Blend compositions

Blend- $0.05 \% \quad 18 \pm 3 \quad 258 \pm 23 \quad 12 \pm 2$

$\begin{array}{llll}\text { Blend }-0.10 \% & 19 \pm 5 & 254 \pm 301 & 14 \pm 4\end{array}$

Blend- $0.50 \% \quad 18 \pm 6 \quad 226 \pm 40 \quad 14 \pm 5$

Blend-1.0\% $20 \pm 4 \quad 244 \pm 25 \quad 15 \pm 7$

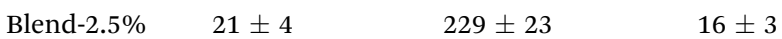


higher total deformation. As the nanoparticles' concentration rises, for low concentrations, Young's modulus increases until a maximum and then decreases (like many nanocomposites). But unlike many nanocomposites, the total deformation does not decrease, and the concentrated formulations showed much higher total strain than the pristine PU. In Fig. 3d, the stressstrain break points are highlighted and it is visible the behavior difference between lowly and highly concentrated Mag@PB $\mathrm{PB}_{1000} /$ PU nanocomposites. .,24,27,28 $^{\mathbf{9}}$

Interestingly, the non-filled compositions also present same rubbery behavior with gain in the elastic modulus value, in the same proportion as each corresponding nanocomposite composition, but show decrease in total strain relative to the PU composition. The stress-strain curves of the non-filled formulations (presented in ESI Fig. S5c $\dagger$ ) become steeper and shortened, compared to the pristine PU, but with lesser tensile strength than the correspondent nanocomposite formulations. In ESI Fig. S5d $\dagger$ we can see a clear difference between the mechanical behavior of the three groups. Although the nonfilled compositions present higher tensile strength than the pristine PU (with loss in total deformation), they have inferior tensile strength and total strain than the nanocomposite compositions. In the case of the highly concentrated compositions, the PU-60\% and PU-70\% compositions, despite lower elastic modulus, showed double the strain with no or little loss in tensile strength compared to the pristine PU.

After an optimum nanoparticle concentration in the low concentration spectrum, as the nanoparticles' concentration rises, the physical aspect of the organic shell becomes more dominant, the Young's modulus and tensile strength values decrease, and the total deformation rises.

However, after the second tensile curve stage, at a given strain, the non-filled formulations present higher stress values than the correspondent nanocomposite formulation. This means the non-filled materials have higher local modulus at these strain values, which can be seen in ESI Fig. $\mathrm{S} 6, \uparrow$ that presents the higher-strain modulus at a given strain. Since the non-filled curves are steeper than the NC's only after the second stage, it would mean that the blend formulations have more gain in restrictions in soft domain deformation.

These results would indicate that the presence of hydroxyl groups of the organic shell cause the increase in the elastic modulus value, not the nanoparticles' inorganic cores. The hydroxyl groups would have reacted with the NCO groups in the prepolymer system and behaved as a crosslinker. The non-filled compositions differ from the nanocomposites in the manner the oligomer poly(1,4-butanediol) is dispersed in the matrix. In the first case, the molecules are randomly distributed within the matrix and have two available hydroxyl groups (on both chain ends) to bond covalently with the PU matrix. In the latter, the molecules are chemically bonded to the inorganic core by one chain end, meaning there is only one hydroxyl group available to interact with the matrix and the molecules are restrained to a brush like conformation around the $\mathrm{Fe}_{3} \mathrm{O}_{4}$ core. Even though the molecules are at molten state, they are pinned to specific points within the matrix - i.e. the cores - which means they offer higher mechanical restraint in this configuration than as randomly distributed molecules within a blend.

Due to the poly(1,4-butanediol) brush like conformation, functional groups and mobility, the hybrid nanoparticles have good interaction with the PU matrix, through covalent hydrogen bonds and mechanical entanglement between molecules.

The stiffening is warranted by the hydroxyl groups at the end of the polymer chains of the organic shell, and the deformability is given by the physical state of the shell. For the highly concentrated compositions, the physical aspect is stronger, and the molten state dominates the mechanical property, decreasing modulus - even though there was "crosslinking" between matrix and nanoparticles - and increasing considerably the total deformation. There is a balance between both functionalities and when one aspect surpasses the other, the mechanical behavior changes: at lower NP concentration, the chemical aspect is stronger, resulting in materials with higher moduli and tensile strength; on the other hand, at very high NP concentration levels, the physical aspect takes over and the resulting material is highly flexible and deformable.

Contrary to typical nanocomposites, where the elastic modulus increases very rapidly with the increase of inorganic volume fraction, these nanocomposites can't be described by current modeling theories, because the inorganic core's effect is strongly affected by the organic shell's chemical and physical aspects, that is, the nanocomposites' properties are dictated by the interphase. ${ }^{9}$

\section{Conclusions}

It was possible to produce evenly dispersed $\mathrm{Fe}_{3} \mathrm{O}_{4} @ P o l y(1,4-$

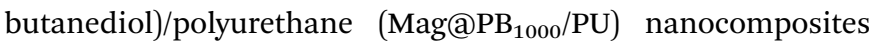
with ease from mixing and casting, with low and high concentrations, ranging from $0.05 \%$ wt to as high as $90 \%$ wt in nanoparticles.

The nanocomposite's final properties (mechanical and thermal) are strongly related to the organic shell's structure, rather than the inorganic core's contribution, indicating that the properties of the nanocomposites are dictated by the interphase between nanoparticle and polymer matrix.

The inorganic portion did not contribute significantly to the compositions' stiffening, as its effects were influenced by the chemical and physical functionalities of the nanoparticles' organic shell. This means that the nanocomposites' behavior is determined by the interphase between the NPs' organic shell and the PU matrix, where hydrogen bonds and mechanical entanglement between molecules occur. For the highly concentrated compositions, PU-60\%, PU-70\% and PU$90 \%$, it seems that the matrix is saturated and the nanoparticles interact mostly with each other, thus decreasing the elastic modulus but allowing larger total strain. The molecules' brush like conformation, the available hydroxyl groups at the chain end and its molten state at room temperature

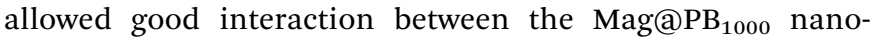
particles and PU matrix, through hydrogen bonds and mechanical entanglements. 


\section{Experimental section}

Materials

Iron(III) acetylacetonate and Terathane ${ }_{1000}{ }^{\circledR} \quad(\operatorname{poly}(1,4-$ butanediol) with molar weight 1000) were purchased from Sigma-Aldrich. Acetone P.A. grade was purchased from Synth, acetonitrile from PANREAC and THF from Tedia. The polyurethane adhesive Adcote $555 \AA$ was received as donation from The Dow Chemical Company.

\section{Synthesis of $\mathrm{Fe}_{3} \mathrm{O}_{4} @ P o l y(1,4$-butanediol)}

Mag@PB 1000 core-shell nanoparticles were synthesized according to synthesis developed by the research group. ${ }^{19}$ Synthesis occurred under nitrogen gas atmosphere and agitation. A volumetric flask with iron(III) acetylacetonate (60 g) and Terathane $_{1000}{ }^{\circledR}(500 \mathrm{~mL})$ was heated up to $110{ }^{\circ} \mathrm{C}$ for 30 minutes, then heated up to $200{ }^{\circ} \mathrm{C}$ for 30 minutes and finally brought to $260{ }^{\circ} \mathrm{C}$ for 90 minutes. After cooling, another batch of iron(III) acetylacetonate $(60 \mathrm{~g})$ was added and the mixture was submitted once more to the previous heating profile. The mixture was washed with a $3: 1$ acetone-acetonitrile solution to remove solvent excess and the nanoparticles were separated with the aid of a magnet. Finally, the synthesized nanoparticles were dispersed in THF solvent.

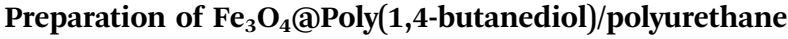 nanocomposites and non-filled formulations}

The nanocomposites were prepared by simple mixing and solvent evaporation. The nanoparticles dispersion was added to the commercial PU and mixed for 10 minutes. The mixture rested for 10 minutes (to allow any bubbles to vanish) and then it was poured into a glass mold and left at room temperature for 24 hours for solvent evaporation. The nanocomposite films were put into a furnace at $60{ }^{\circ} \mathrm{C}$ for 24 hours and then they were subjected to vacuum to eliminate any solvent residue. The compositions' NP's weight percentage varied from $0.05 \%$ to $90 \%$ and were called PU-0.05\%, PU-0.10\%, PU-0.50\%, PU-1.0\%, PU2.5\%, PU-5.0\%, PU-60\%, PU-70\% and PU-90\%. Additional THF solvent was added to compositions from $0 \%$ wt to $2.5 \%$ wt to match the PU-solvent ratio of the PU-5.0\% composition, so that all formulations presented the same conditions during curing. Non-filled compositions were also prepared to evaluate the organic layer's contribution on the thermomechanical properties of the PU. Named Blend-0.05\%, Blend-0.10\%, Blend- $0.50 \%$, Blend-1.0\% and Blend-2.5\%, they consist of blends of PU and processed poly(1,4-butanediol) in the same proportions found in the nanocomposites PU-0.05\% through $\mathrm{PU}-2.5 \%$ and also have additional THF solvent added to match PU-solvent ratio of the nanocomposite's formulations. The processed poly $(1,4-$ butanediol) consists of Therathane ${ }_{1000}{ }^{\circledR}$ that was subjected to the same heating profile of the nanoparticles' synthesis.

\section{Characterization}

Thermal stability and organic mass percentage of the nanoparticles were evaluated by TGA on TA Instruments Q50 equipment, under inert nitrogen atmosphere and at $10{ }^{\circ} \mathrm{C} \mathrm{min}^{-1}$ heating rate. TEM imaging allowed measurement of size and size distribution of the nanoparticles' cores and nanocomposites morphology observation. DSC analysis, done in TA Q100 equipment with 10 and $20{ }^{\circ} \mathrm{C} \mathrm{min}{ }^{-1}$ heating rate from $-80{ }^{\circ} \mathrm{C}$ to $150{ }^{\circ} \mathrm{C}$, showed the thermal behavior of the nanoparticles, nanocomposites and blends. Tensile test was performed on Instron equipment, at a $500 \mathrm{~mm} \mathrm{~min}^{-1}$ traction speed at room temperature (see details in moduli measurement in $\mathrm{ESI} \dagger$ ). Based on ASTM D412 standard, at least 10 specimens in dumbbell shape were prepared for the low concentration nanocomposite and blend formulations, and at least 5 specimens were prepared in rectangular shape for the highly concentrated nanocomposite formulations.

\section{Conflicts of interest}

There are no conflicts to declare.

\section{Acknowledgements}

The authors would like to thank FAPESP, CNPq and CAPES foundations for funding the research: grant no. 2016/13191-7, São Paulo Research Foundation (FAPESP); grant no. 2013/ 07296-2, Centro de Pesquisa, Inovação e Difusão/FAPESP (CEPID/FAPESP); grant no. 131365/2016-9, National Council for Scientific and Technological Development (CNPq); and Finance Code 001, Coordenação de Aperfeiçoamento de Pessoal de Nível Superior - Brasil (CAPES). The authors also thank the Brazilian Nanotechnology National Laboratory (LNNanoCNPEM) and Laboratory of Structural Characterization (LCE/ DEMa/UFSCar) for the microscopy facilities.

\section{Notes and references}

1 D. R. Paul and L. M. Robeson, Polymer, 2008, 49, 3187-3204. 2 H. Zou, S. Wu and J. Shen, Chem. Rev., 2008, 108, 3893-3957. 3 J. R. Potts, D. R. Dreyer, C. W. Bielawski and R. S. Ruoff, Polymer, 2011, 52, 5-25.

4 R. Mangal, S. Srivastava and L. A. Archer, Nat. Commun., 2015, 6, 1-9.

5 S. M. Liff, N. Kumar and G. H. McKinley, Nat. Mater., 2007, 6, 76-83.

6 N. J. Fernandes, H. Koerner, E. P. Giannelis and R. A. Vaia, MRS Commun., 2013, 3, 13-29.

7 L. S. Schadler, S. K. Kumar, B. C. Benicewicz, S. L. Lewis and S. E. Harton, MRS Bull., 2007, 32, 335-340.

8 T. Hanemann and D. V. Szabó, Materials, 2010, 3, 3468-3517. 9 S. K. Kumar, N. Jouault, B. Benicewicz and T. Neely, Macromolecules, 2013, 46, 3199-3214.

10 A. Halperin, M. Tirrell and T. P. Lodge, Macromol. Synth. Order Adv. Prop., 1992, vol. 100, pp. 31-71.

11 Y. Chen, Macromolecules, 2012, 45, 2619-2631.

12 S. Anaya, B. Serrano, B. Herrero, A. Cervera and J. Baselga, ACS Appl. Mater. Interfaces, 2014, 6, 14460-14468.

13 J. Choi, C. M. Hui, J. Pietrasik, H. Dong, K. Matyjaszewski and M. R. Bockstaller, Soft Matter, 2012, 8, 4072-4082. 
14 A. B. Bourlinos, S. R. Chowdhury, D. D. Jiang, Y. U. An, Q. Zhang, L. A. Archer and E. P. Giannelis, Small, 2005, 1, 80-82.

15 P. Rittigstein, R. D. Priestley, L. J. Broadbelt and J. M. Torkelson, Nat. Mater., 2007, 6, 278-282.

16 M. Arslan, M. Tasdelen, M. Arslan and M. A. Tasdelen, Polymer, 2017, 9, 499.

17 M. Giovino, J. Pribyl, B. Benicewicz, S. Kumar and L. Schadler, Polymer, 2017, 131, 104-110.

18 H. Xin, S. Jun, W. Ming, A. Latif and J. Lim, Colloids Surf., A, 2018, 539, 209-220.

19 R. H. Gonçalves, C. A. Cardoso and E. R. Leite, J. Mater. Chem., 2010, 20, 1167.

20 A. Mohammadi, M. Barikani and M. Barmar, J. Mater. Sci., 2013, 48, 7493-7502.

21 S. Kango, S. Kalia, A. Celli, J. Njuguna, Y. Habibi and R. Kumar, Prog. Polym. Sci., 2013, 38, 1232-1261.
22 P. G. de Gennes, Macromolecules, 1980, 13, 1069-1075.

23 O. V. de Oliveira, L. T. Costa and E. R. Leite, Comput. Theor. Chem., 2016, 1092, 52-56.

24 W. Wang and J. Wu, J. Appl. Polym. Sci., 2018, 135, 1-10.

25 H. W. Engels, H. G. Pirkl, R. Albers, R. W. Albach, J. Krause, A. Hoffmann, H. Casselmann and J. Dormish, Angew. Chem., Int. Ed., 2013, 52, 9422-9441.

26 F. Yeh, B. S. Hsiao, B. B. Sauer, S. Michel and H. W. Siesler, Macromolecules, 2003, 36, 1940-1954.

27 D. Maillard, S. K. Kumar, B. Fragneaud, J. W. Kysar, A. Rungta, B. C. Benicewicz, H. Deng, L. C. Brinson and J. F. Douglas, Nano Lett., 2012, 12, 3909-3914.

28 A. Haase, P. Hesse, L. Brommer, O. Jacobs, C. Abetz, U. A. Handge, A. Boschetti-De-Fierro and V. Abetz, Macromol. Mater. Eng., 2013, 298, 292-302. 